\title{
MEASUREMENT OF THE APS STORAGE RING ELECTRON BEAM ENERGY SPREAD USING UNDULATOR SPECTRA*
}

\author{
B. X. Yang and J. J. Xu ${ }^{\dagger}$ \\ Advanced Photon Source, Argonne National Laboratory, Argonne, IL
}

\begin{abstract}
The angle-integrated flux spectrum of an undulator has sharp dropoffs at harmonic photon energies. Its derivative therefore shows pronounced peaks with rms width $\Delta \omega / \omega$ $\sim 0.4 / \mathrm{nN}$, where $\omega$ is the photon energy, $n$ is the harmonic number, and $N$ is the number of undulator periods. For the fundamental photon energy of the Advanced Photon Source diagnostics undulator, the derivative of the angleintegrated flux spectrum is a peak with rms width of $\sim 0.2 \%$. By using this feature, we have successfully developed techniques to measure the energy centroid, spread, and momentum compaction factor of the APS storage ring beam. In this paper, we present analytical and numerical analyses of the undulator spectrum derivative and examine the sources of experimental errors of this technique.
\end{abstract}

\section{INTRODUCTION}

Non-invasive measurements of the beam energy and rms energy spread are essential for the study of electron longitudinal beam dynamics. In storage rings, the size of a fully damped beam is given by the following

$$
\sigma_{x}^{2}=\beta_{x} \varepsilon_{x}+\left(\eta_{x} \delta\right)^{2}
$$

where $\beta_{x}$ is the beta function, $\eta_{x}$ is the dispersion function at the source point, $\varepsilon_{x}$ is the horizontal emittance, and $\delta=\sigma_{E} / E$, the relative rms energy spread of the beam [1]. The beam energy measurements are normally performed with simultaneous measurements of beam sizes at high and low dispersive locations of the magnetic lattice. The accuracy of the beam energy measurements is often limited by that of the lattice parameters, $\beta_{x}$ and $\eta_{x}$, which are difficult to measure with high accuracy.

In 1996, Tarazona and Elleaume used undulator radiation spectra to derive electron beam energy and emittance by fitting the experimental spectra to a straightforward theoretical model [2]. In that work, the dispersion function at the source was no longer required. But a good knowledge of the beta function was still needed.

In this work, we propose a new measurement using the undulator radiation that employs a different part of the undulator spectrum (measuring the angle-integrated spectrum in a narrow energy span near undulator harmonics) and a different procedure for data treatment (compare the derivative of the spectrum with the model calculation).

We introduce the basics of the technique and discuss analytical expressions in Section 2. In Section 3, we propose experimental schemes to measure the electron beam energy spread and absolute energy. Sources of

\footnotetext{
* Work supported by U.S. Department of Energy, Office of Basic Energy Sciences, under Contract No. W-31-109-ENG-38.

†xujjk@online.sh.cn, Fudan University, Shanghai, China
}

experimental errors and possible single-bunch single-pass measurement are also discussed.

\section{BASIC FORMULAE}

\subsection{Angular Distribution of Undulator Radiation}

The angular distribution of undulator radiation (within a spectral width $\Delta \omega)$ is given by the following [3],

$$
\frac{d F_{\pi, \sigma}^{(0)}}{d \Omega}=\alpha \frac{\Delta \omega}{\omega} \frac{I}{e}\left(\frac{\gamma N p}{2}\right)^{2} S_{N}(\omega, \theta) B_{\pi, \sigma}{ }^{2}
$$

where $\alpha$ is the fine structure constant, $\omega$ the photon frequency, $K$ the undulator parameter, $N$ the number of undulator periods, $E=\gamma m c^{2}$ the energy of the electron, and $(\theta, \varphi)$ are polar angles. The superscript $(0)$ means that the expression is for electron beams with zero emittance and zero energy spread. The fundamental resonance photon energy is

$$
\omega_{1}(\gamma, \theta)=\frac{2 \pi}{\lambda_{u}} \frac{2 \gamma^{2}}{1+\frac{K^{2}}{2}+\gamma^{2} \theta^{2}},
$$

where $\lambda_{u}$ is the undulator period length. The functions $S_{N}$ and $B$ are given by

$$
S_{N}=\left[\sin \frac{N \pi \omega}{\omega_{1}(\gamma, \theta)} / N \sin \frac{\pi \omega}{\omega_{1}(\gamma, \theta)}\right]^{2}
$$

and

$$
\left(\begin{array}{l}
B_{\pi} \\
B_{\sigma}
\end{array}\right)=\frac{1}{\pi} \int_{-\pi}^{\pi}\left(\begin{array}{c}
\frac{\gamma \theta \cos \varphi}{K}-\cos \xi \\
\frac{\gamma \theta \sin \varphi}{K}
\end{array}\right) e^{i\left(\frac{\omega}{\omega_{1}(\theta)} \xi-p \gamma \theta \cos \varphi \sin \xi+q \sin 2 \xi\right)} d \xi,
$$

where $p=2 \frac{\omega}{\omega_{1}(\gamma, 0)} \frac{K}{1+K^{2} / 2}$, and $q=\frac{1}{4} \frac{\omega}{\omega_{1}(\gamma, 0)} \frac{K^{2}}{1+K^{2} / 2}$

\subsection{Angle-Integrated Spectrum of Undulator Radiation for Mono-Energetic Electron Beam}

Integrating over all angles, we obtain the angleintegrated spectrum

$$
F_{\pi, \sigma}^{(0)}=\frac{\alpha N^{2} \gamma^{2} p^{2}}{4} \frac{\Delta \omega}{\omega} \frac{I}{e} \int_{0}^{\pi} S_{N}(\omega, \theta) \sin \theta d \theta \int_{0}^{2 \pi} B_{\pi, \sigma}{ }^{2} d \varphi
$$

Let us define a dimensionless frequency $v$ and a new angle variable $\mu$,

$$
v=\frac{\omega}{\omega_{1}(\gamma, 0)}, \quad \mu=\frac{\omega}{\omega_{1}(\gamma, \theta)}=v\left(1+\frac{\gamma^{2} \theta^{2}}{1+\frac{K^{2}}{2}}\right) .
$$

We have

$$
S_{N}=S_{N}(\mu)=\left[\frac{\sin N \mu \pi}{N \sin \mu \pi}\right]^{2}
$$


and

$$
F_{\pi, \sigma}^{(0)}=\frac{\alpha N^{2}}{2} \frac{\Delta \omega}{\omega_{1}(\gamma, 0)} \frac{I}{e} \frac{K^{2}}{1+K^{2} / 2} \int_{v}^{\infty} S_{N}(\mu) d \mu \int_{0}^{2 \pi} B_{\pi, \sigma}{ }^{2} d \varphi,
$$

where we have used the exact expression $\mu(\theta=0)=v$ and the approximation $\mu(\theta=\pi) \rightarrow \infty$. For this work, we are very interested in the scaled derivative of the total flux spectrum, defined as

$$
D_{\pi, \sigma}^{(0)}(\gamma, v) \equiv-v \frac{d F_{\pi, \sigma}^{(0)}}{d v} .
$$

Because $\mu$ is a dummy variable in the integration and is independent of $v$, we can write the total angle-integrated radiation spectrum for photons of both polarizations,

$$
\begin{aligned}
D^{(0)}(\gamma, v) & \equiv D_{\pi}^{(0)}(\gamma, v)+D_{\sigma}^{(0)}(\gamma, v) \\
& =\sin ^{2} \frac{v \pi}{2} H^{(0)}(\gamma, v) S_{N}(v)+C^{(0)}(\gamma, v)
\end{aligned},
$$

where

$$
H^{(0)}(\gamma, v)=4 \pi \alpha N^{2} \frac{\Delta \omega}{\omega_{1}(\gamma, 0)} \frac{I}{e} q\left[\mathbf{J}_{\frac{v-1}{2}}(q)-\mathbf{J}_{\frac{v+1}{2}}(q)\right]^{2},
$$

and

$$
\begin{aligned}
& C^{(0)}(\gamma, v) \equiv C_{\pi}^{(0)}(\gamma, v)+C_{\sigma}^{(0)}(\gamma, v) \\
& =-2 \pi \alpha q N^{2} \frac{\Delta \omega}{\omega_{1}(\gamma, 0)} \frac{I}{e} \int_{v}^{\infty} d \mu S_{N}(\mu) \int_{0}^{2 \pi} \frac{\partial\left(B_{\pi}{ }^{2}+B_{\sigma}{ }^{2}\right)}{\partial v} d \varphi
\end{aligned}
$$

$\mathbf{J}_{v}(\mathrm{q})$ is Auger function. Because both $H$ and $C$ are slow functions of $v$, the spectral derivative is dominated by the sinc function $S_{N}(v)$, with its peak at the nominal harmonic energies. This feature can be clearly seen in Fig. 1.

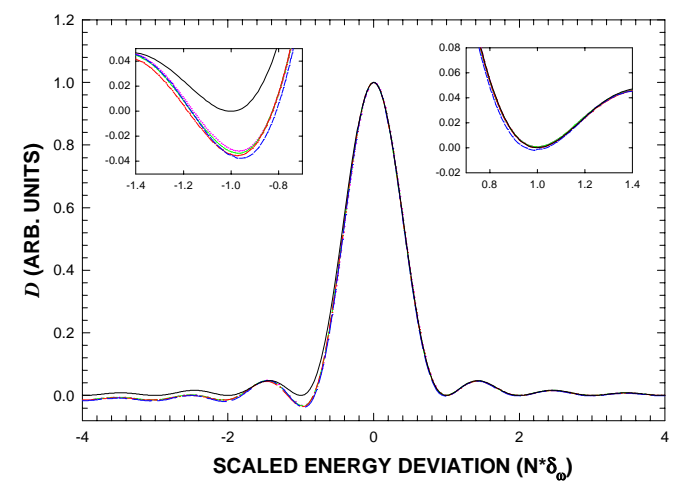

Figure 1: Calculated derivative spectra for the APS diagnostics undulator $(N=198)$. All spectra were normalized to have the same maximum value. The dotted line is for $K=0.01$, the dashed line for $K=0.1$, the dashdot line for $K=0.5$, and the long-dashed line for $K=1.0$. The solid line (top) is the sinc function. Its center peak has an rms width of $0.36 / n N$.

\subsection{Electron Beam With Finite Energy Spread}

Let us consider electron beams with Gaussian energy distribution,

$$
\rho(\delta)=\frac{1}{\sqrt{2 \pi} \sigma_{\delta}} e^{-\frac{\delta^{2}}{2 \sigma_{\delta}^{2}}}
$$

where the relative momentum change is defined as $\delta=\Delta E / E_{0}=\Delta \gamma / \gamma_{0}$, and $E_{0}$ is the centroid energy. Using the new variables defined in Eq. (7) and the following

$$
v_{0}=\frac{\omega}{\omega_{1}\left(\gamma_{0}, 0\right)},
$$

we have $v=v_{0}(1-2 \delta)$. Convolution of Eq. (15) and (6) leads to the angle-integrated photon flux. After dropping higher orders of $\delta$, we obtain the derivative of the spectrum in the form of a convolution integral,

$$
D_{\pi, \sigma}\left(\gamma_{0}, v_{0}\right)=\frac{1}{\sqrt{2 \pi} \sigma_{\delta}} \int_{-\infty}^{\infty} e^{-\frac{\delta^{2}}{2 \sigma_{\delta}^{2}}} D_{\pi, \sigma}^{(0)}\left(\gamma_{0}, n\left(1+\delta_{\omega}-2 \delta\right)\right) d \delta,
$$

where $\delta_{\omega}=\Delta \omega / \omega_{0}=n \Delta v$ is the relative photon energy deviation.

\section{BEAM ENERGY MEASUREMENTS}

Figure 2 shows the schematics of an experimental setup for measuring angle-integrated flux spectrum. A rotating crystal selects the $\mathrm{x}$-ray energy according to Bragg's law, $\lambda=2 d \sin \theta$, where $\theta$ is the incidence angle of the x-ray beam. The reflected x-rays are collected by an integrating detector. The relative photon energy change is given by $\Delta \omega / \omega_{0}=-\Delta \lambda / \lambda_{0}=-\Delta \theta / \tan \theta_{0}$, where $\theta_{0}$ is the angle at the center of the flux jump. Converting it to the equivalent electron energy deviation, we have

$$
\frac{\Delta \gamma}{\gamma_{0}}=\frac{\Delta \omega}{2 \omega}=-\frac{\Delta \theta}{2 \tan \theta} .
$$

The experimentally measured flux curve, $F(\theta)$, can be differentiated numerically to obtain the scaled spectral derivative.

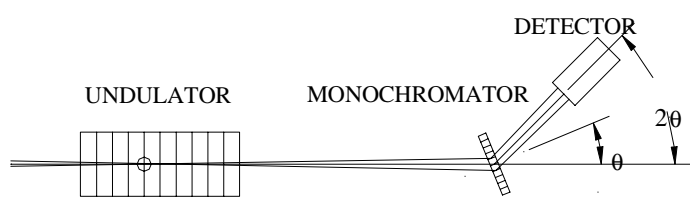

Figure 2: Schematic of the spectrum measurement of angle-integrated flux. The monochromator crystal uses Laue reflection with a Bragg angle $\theta$, and the detector is supported by a second rotary stage following $2 \theta$.

\subsection{Electron Beam Energy Spread Measurement}

The width obtained in fitting the experimentally measured spectral derivative to a Gaussian peak is a sum,

$$
\sigma_{\exp }^{2}=\sigma_{\delta}^{2}+\sigma_{\text {res }}^{2} \text {. }
$$

where the total resolution is the sum of contributions from various sources

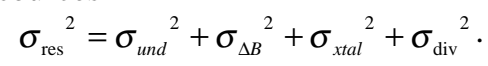

Table 1 explains the meaning of these terms and lists their typical values at the APS storage ring. The ideal resolution dominates in the case of the APS undulator. It is also significant that the electron beam size has no impact on the energy measurement. 
The contribution of the undulator field error is difficult to express in closed forms. For nearly perfect undulators, the trajectory error does not affect the angle-integrated spectrum to the first order, so we estimate,

$$
\sigma_{\Delta B} \approx \frac{0.18}{n N} \sigma_{\Delta n}=\frac{0.18}{N} \sigma_{\Delta},
$$

where $\sigma_{\Delta}$ and $\sigma_{\Delta n}$ are the rms phase errors of the fundamental and $n$-th harmonic.

Table 1 Sources of Experimental Error / Resolution

\begin{tabular}{|c|l|c|}
\hline Expression & \multicolumn{1}{|c|}{ Source } & $\begin{array}{c}\text { Typical } \\
\text { values }\left(10^{-3}\right)\end{array}$ \\
\hline$\sigma_{\text {und }} \approx \frac{0.18}{n N}$ & $\begin{array}{l}\text { Ideal resolution: SINC } \\
\text { function central peak }\end{array}$ & 0.9 \\
\hline$\sigma_{\Delta B} \approx \frac{0.18}{N} \sigma_{\Delta}$ & Undulator field error & $<0.08$ \\
\hline$\sigma_{x t a l} \approx \frac{\Delta \theta_{x t a l}}{6 \tan \theta}$ & $\begin{array}{l}\text { Darwin width, mosaic } \\
\text { angle and thermal } \\
\text { distortion of the crystal }\end{array}$ & $<0.06$ \\
\hline$\sigma_{d i v} \approx \frac{\sigma_{x^{\prime}}}{2 \tan \theta}$ & $\begin{array}{l}\text { Beam divergence / } \\
\text { angular motion }\end{array}$ & $<0.1(x)$ \\
\hline
\end{tabular}

$*$ We assume $n N=200, \theta(\operatorname{Si}[200])=7.21^{\circ}, \sigma_{\Delta}<5^{\circ}, \Delta \theta_{\text {xtal }}$ $<10$ arc-sec, $\sigma_{x^{\prime}}<25 \mu \mathrm{rad}$, and $\sigma_{y^{\prime}}<5 \mu \mathrm{rad}$.

\subsection{Absolute Electron Beam Energy Measurement}

Equation (11) indicates that the spectral derivative peaks very nearly at the nominal harmonic energy. This can be used to estimate the beam energy with the center angle obtained in fitting the experimentally measured spectral derivative, $\theta_{0}$.

$$
\gamma^{2}=\frac{\lambda_{u}}{4 d \sin \theta_{0}}\left(1+\frac{K^{2}}{2}\right) .
$$

The error in the beam energy can therefore be given by

$$
\left(\frac{\Delta \gamma}{\gamma}\right)^{2}=\frac{\left(\frac{\Delta \lambda_{u}}{\lambda_{u}}\right)^{2}+\left(\frac{K^{2} \frac{\Delta K}{K}}{1+\frac{K^{2}}{2}}\right)^{2}+\left(\frac{\Delta d}{d}\right)^{2}+\left(\frac{\Delta \theta_{0}}{\theta_{0}}\right)^{2}}{4} .
$$

The errors from the undulator can be minimized when running at low power $(K)$. Errors from the monochromator crystal can be minimized when a silicon crystal is used at cryogenic temperatures [4]. The difficulty of measuring the absolute value of the angle $\theta$ can be overcome by scanning the monochromator through spectral peaks at both negative and positive angles and taking the difference as $2 \theta_{0}$ (Fig. 3). Table 2 lists the typical values at the APS storage ring. Under these conditions, an accuracy of $10^{-4}$ can be obtained for the absolute energy measurement.

Turn-by-turn energy measurements can be performed when flux at four crystal angles $\left(\theta_{1}\right.$ though $\theta_{4}$ in Fig. 3$)$ is obtained simultaneously with Laue crystal arrays.

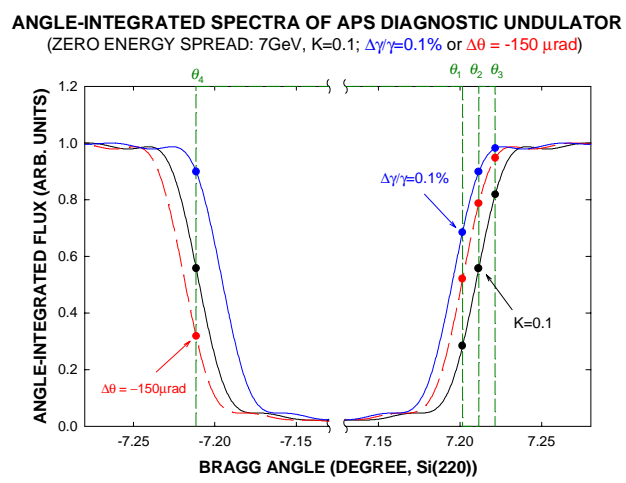

Figure 3: Calculated angle-integrated spectrum of the APS diagnostics undulator as a function of Bragg angle of $\mathrm{Si}(220)$. The figure shows the use of a negative angle to calibrate the absolute angle offset.

Table 2 Sources of Experimental Error / Resolution

\begin{tabular}{|c|l|c|}
\hline Expression & \multicolumn{1}{|c|}{ Source } & $\begin{array}{c}\text { Typical } \\
\text { values }\left(10^{-3}\right)\end{array}$ \\
\hline$\frac{\Delta \lambda_{u}}{\lambda_{u}}$ & $\begin{array}{l}\text { Error in undulator period } \\
\text { length }\end{array}$ & 0.11 \\
\hline$K^{2} \frac{\Delta K}{K}$ & $\begin{array}{l}\text { Undulator field } \\
\text { uncertainty }\end{array}$ & $<0.03$ \\
\hline$\frac{\Delta d}{d} \approx \alpha \Delta T$ & $\begin{array}{l}\text { Thermal expansion of the } \\
\text { crystal lattice }\end{array}$ & $<0.01$ \\
\hline$\frac{\Delta \theta_{0}}{\theta_{0}}$ & Angle measurement error & $<0.05$ \\
\hline
\end{tabular}

* We assume $\lambda_{u}=18 \mathrm{~mm}, \Delta \lambda_{u}=2 \mu \mathrm{m}, K=0.03, \Delta K / K$ $<0.03, \alpha \sim-0.5 \times 10^{-6} \mathrm{~K}^{-1}, \Delta T \sim 20^{\circ} \mathrm{K}$, and $\Delta \theta_{0}<5$ arc-sec.

\section{CONCLUSION}

We proposed to use the angle-integrated undulator spectra to measure the centroid and rms spread of the electron beam energy. The advantages of this new technique are: (1) its calibration is independent of the ring lattice parameter, (2) it is not sensitive to the beam emittance and other lattice parameters, and (3) a fast turnby-turn measurement is feasible.

\section{REFERENCES}

[1] J. Seeman, "Energy Measurement with Electron Beams," in Handbook of Accelerator Physics and Engineering, ed. W. Chao and M. Tigner, World Scientific (1999).

[2] E. Tarazona and P. Elleaume, "Measurement of the Absolute Energy and Energy Spread of the ESRF Electron Beam Using Undulator Radiation," Rev. Sci. Instrum. 67 (1996).

[3] K. J. Kim, "Characteristics of Synchrotron Radiation," AIP Conf. Proc. 184, 565 (1988).

[4] B. Yang, et al, "Performance Analysis of Cryogenic Silicon Laue Monochromators at APS Undulators," SPIE Proc. 1997, 302 (1993). 